\title{
Searches for heavy neutral lepton production and lepton flavour violation in kaon decays at the NA62 experiment
}

\author{
Stoyan Trilov*t \\ University of Bristol \\ E-mail: stovan.trilovdcern.ch
}

\begin{abstract}
Searches for heavy neutral lepton (HNL) production in charged kaon decays using the data collected by the NA62 experiment at CERN are reported. Upper limits are established on the elements of the extended neutrino mixing matrix for heavy neutral lepton mass in the range 130-450 $\mathrm{MeV}$, improving on the results from previous HNL production searches. The status and prospects of searches for lepton flavour and lepton number violation in kaon decays at the NA62 experiment is also presented.
\end{abstract}

The 20th International Workshop on Neutrinos (NuFact2018)

12-18 August 2018

Blacksburg, Virginia

\footnotetext{
*Speaker.

${ }^{\dagger}$ On behalf of the NA62 Collaboration: R. Aliberti, F. Ambrosino, R. Ammendola, B. Angelucci, A. Antonelli, G. Anzivino, R. Arcidiacono, M. Barbanera, A. Biagioni, L. Bician, C. Biino, A. Bizzeti, T. Blazek, B. Bloch-Devaux, V. Bonaiuto, M. Boretto, M. Bragadireanu, D. Britton, F. Brizioli, M.B. Brunetti, D. Bryman, F. Bucci, T. Capussela, A. Ceccucci, P. Cenci, V. Cerny, C. Cerri, B. Checcucci, A. Conovaloff, P. Cooper, E. Cortina Gil, M. Corvino, F. Costantini, A. Cotta Ramusino, D. Coward, G. D’Agostini, J. Dainton, P. Dalpiaz, H. Danielsson, N. De Simone, D. Di Filippo, L. Di Lella, N. Doble, B. Dobrich, F. Duval, V. Duk, J. Engelfried, T. Enik, N. Estrada-Tristan, V. Falaleev, R. Fantechi, V. Fascianelli, L. Federici, S. Fedotov, A. Filippi, M. Fiorini, J. Fry, J. Fu, A. Fucci, L. Fulton, E. Gamberini, L. Gatignon, G. Georgiev, S. Ghinescu, A. Gianoli, M. Giorgi, S. Giudici, F. Gonnella, E. Goudzovski, C. Graham, R. Guida, E. Gushchin, F. Hahn, H. Heath, T. Husek, O. Hutanu, D. Hutchcroft, L. Iacobuzio, E. Iacopini, E. Imbergamo, B. Jenninger, K. Kampf, V. Kekelidze, S. Kholodenko, G. Khoriauli, A. Khotyantsev, A. Kleimenova, A. Korotkova, M. Koval, V. Kozhuharov, Z. Kucerova, Y. Kudenko, J. Kunze, V. Kurochka, V.Kurshetsov, G. Lanfranchi, G. Lamanna, G. Latino, P. Laycock, C. Lazzeroni, M. Lenti, G. Lehmann Miotto, E. Leonardi, P. Lichard, L. Litov, R. Lollini, D. Lomidze, A. Lonardo, P. Lubrano, M. Lupi, N. Lurkin, D. Madigozhin, I. Mannelli, G. Mannocchi, A. Mapelli, F. Marchetto, R. Marchevski, S. Martellotti, P. Massarotti, K. Massri, E. Maurice, M. Medvedeva, A. Mefodev, E. Menichetti, E. Migliore, E. Minucci, M. Mirra, M. Misheva, N. Molokanova, M. Moulson, S. Movchan, M. Napolitano, I. Neri, F. Newson, A. Norton, M. Noy, T. Numao, V. Obraztsov, A. Ostankov, S. Padolski, R. Page, V. Palladino, C. Parkinson, E. Pedreschi, M. Pepe, M. Perrin-Terrin, L. Peruzzo, P. Petrov, F. Petrucci, R. Piandani, M. Piccini, J. Pinzino, I. Polenkevich, L. Pontisso, Yu. Potrebenikov, D. Protopopescu, M. Raggi, A. Romano, P. Rubin, G. Ruggiero, V. Ryjov, A. Salamon, C. Santoni, G. Saracino, F. Sargeni, V. Semenov, A. Sergi, A. Shaikhiev, S. Shkarovskiy, D. Soldi, V. Sougonyaev, M. Sozzi, T. Spadaro, F. Spinella, A. Sturgess, J. Swallow, S. Trilov, P. Valente, B. Velghe, S. Venditti, P. Vicini, R. Volpe, M. Vormstein, H. Wahl, R. Wanke, B. Wrona, O. Yushchenko, M. Zamkovsky, A. Zinchenko
} 


\section{1. $v \mathbf{M S M}$}

Massive neutrinos are not accounted for in the standard model (SM). This necessitates an extension to the SM explaining the experimental observation of neutrinos having mass. One such framework is known as the neutrino minimal standard model or $v$ MSM [W], where right-handed neutrinos are introduced. If the mass of these heavy neutral leptons (HNL), $m_{N}$, satisfies the criterion: $m_{N}<m_{K}-m_{l}$ where $l=e, \mu$, then these sterile neutrinos can be produced via the process:

$$
\Gamma\left(K^{ \pm} \rightarrow l^{ \pm} N\right)=\Gamma\left(K^{ \pm} \rightarrow l^{ \pm} v_{l}\right) \cdot \rho_{m_{N}} \cdot\left|U_{l 4}\right|^{2},
$$

in which $U_{l 4}$ is the extended neutrino mixing matrix, and $\rho_{m_{N}}$ is a factor accounting for the kinematic phase space and helicity suppression.

\section{HNL searches}

The search for heavy neutrinos presented here is based on a 2015 data sample, consisting of around $1.2 \times 10^{3}$ Super Proton Synchrotron (SPS) spills. The analysis strategy consists of searching for a peak in the missing mass spectrum, $m_{\text {miss }}^{2}=\left(P_{K}-P_{l^{+}}\right)^{2}$, where the electron/muon modes are known as $K_{e 2} / K_{\mu 2}$ respectively. The incoming kaons are tagged by the kaon tagger (KTAG) [■], and their momenta are measured using fully reconstructed $K^{+} \rightarrow \pi^{+} \pi^{+} \pi^{-}$decays, whereas the daughter particle momentum is provided by the STRAW magnetic spectrometer. The analysis proceeds via a common one track selection, where exactly one good track is required in the final state. Events with photons are vetoed using the photon veto detectors, with distinct systems detecting photons emitted at small, medium, and large angles. Following the common selection, particle identification (PID) is performed using a ring-imaging Čerenkov (RICH) counter, two hadronic calorimeters (MUV1/2), a plastic scintillator muon veto (MUV3), and an electromagnetic calorimeter (LKr).

The above selection yields $1767 K_{e 2}$ candidates and $24 \mathrm{M} K_{\mu 2}$ candidates in the SM region of $m_{m i s s}^{2}=0$. These yields are equivalent to $(3.01 \pm 0.11) \times 10^{8}$ kaon decays $\left(N_{K}\right)$ for the electron mode, and $N_{K}=(1.06 \pm 0.02) \times 10^{8}$ for the muon mode. This difference in $N_{K}$ comes from the different scaling of the electron and muon trigger lines. The missing mass spectra of the $K_{e 2}$ and $K_{\mu 2}$ candidates are shown in figures $\square$ and $\mathbb{}$ m respectively. The main backgrounds in the $K_{e 2}$ sample originate from $\mu^{+}$decays in-flight, and beam $\pi^{+}$decays with the presence of an accidental in-time kaon in the KTAG. The largest contribution to the background in the $K_{\mu 2}$ sample is radiative SM $K_{\mu 2}$ decays, $K^{+} \rightarrow \mu^{+} v \gamma$, where the photon is outside the geometrical acceptance of the large angle veto (LAV).

A HNL mass scan is performed in the range of $170<m_{N}<448 \mathrm{MeV} / \mathrm{c}^{2}$ for $K_{e 2}$, and $250<$ $m_{N}<373 \mathrm{MeV} / \mathrm{c}^{2}$ for $K_{\mu 2}$, where the width of the window is $1.5 \sigma_{m_{N}}$ (HNL mass resolution). The presence of signal in a particular mass window would be indicated by an excess in the number of observed events $\left(n_{o b s}\right)$ against the number of expected background events $\left(n_{\text {exp }}\right)$. The uncertainties on $n_{o b s}$ are evaluated using a combination of data-driven and Monte Carlo techniques.

\section{Heavy neutral leptons limits and prospects}

In the absence of clear excess in $n_{o b s}$, limits have been set on the number HNL signal events 
$\left(n_{U L}\right)$ using the Rolke-Lopez method [ [ $]$ ]. These can be converted into limits on HNL branching fractions and mixing parameters using the following expressions,

$$
B_{U L}\left(K^{+} \rightarrow l^{+} N\right)=\frac{n_{U L}}{N_{K} \times A\left(m_{N}\right)}, \quad\left|U_{l 4}\right|_{U L}^{2}=\frac{B_{U L}\left(K^{+} \rightarrow l^{+} N\right)}{B_{U L}\left(K^{+} \rightarrow l^{+} v_{l}\right)} \times \frac{1}{\rho_{m_{N}}},
$$

where $A\left(m_{N}\right)$ is the signal acceptance, a function of the heavy neutrino mass, $m_{N}$.

Upper limits are set at the level of $10^{-7}$ for $\left|U_{e 4}\right|^{2}$, and $10^{-6}$ for $\left|U_{\mu 4}\right|^{2}$ for the aforementioned mass ranges [四], shown in figure एव. These results either improve upon or extend current limits, as shown in figure पd. With larger statistics, and improved analysis techniques, e.g. the use of the Gigatracker (GTK) beam spectrometer, $\left|U_{l 4}\right|^{2}$ limits are expected reach the level of $10^{-8}$.

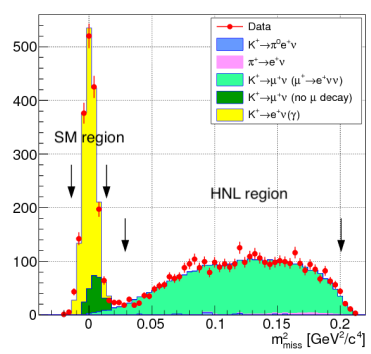

(a)

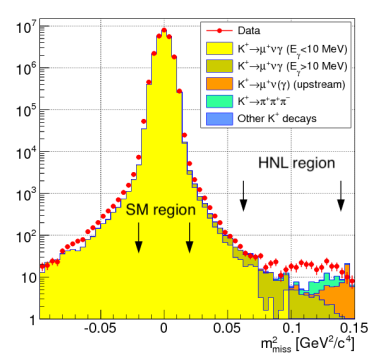

(b)

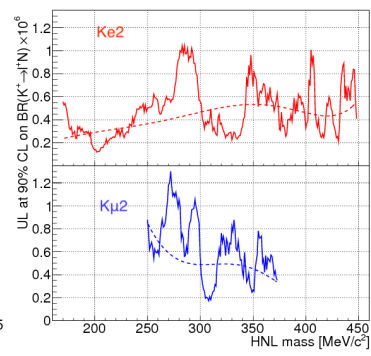

(c)

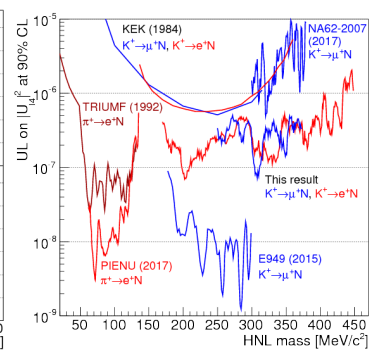

(d)

Figure 1: (a) $m_{m i s s}^{2}$ spectrum for $K_{e 2}$ decays. (b) $m_{m i s s}^{2}$ spectrum for $K_{\mu 2}$. (c) Upper limits set on the branching ratio of the HNL. (d) The new $\left|U_{l 4}\right|^{2}$ upper limits compared against previous results. For (c) and (d), $K_{e 2}$ limits are in red, and $K_{\mu 2}$ limits are in blue.

\section{Lepton flavour(number) violating processes at NA62}

NA62 has a wide physics programme, and is expected to be competitive in a number of lepton flavour(number) violating $(\mathrm{LF}(\mathrm{N}) \mathrm{V})$ channels, reaching sensitivities down to $10^{-11}$. Around $2 \times$ $10^{4} K^{+} \rightarrow \pi^{+} \mu^{+} \mu^{-}$candidates are expected to be collected, making it world's largest sample. That sample would allow the searches for $K^{+} \rightarrow \pi^{+} S, S \rightarrow \mu+\mu^{-}$to reach sensitivities of around $10^{-10}$. Single event sensitivity (SES) for $K^{+} \rightarrow \pi^{-} \mu^{+} \mu^{+}$is expected to reach $2 \times 10^{-11}$, where the measurement is background free.

$K^{+} \rightarrow \pi^{-} e^{+} e^{+}$is another channel whose search for is not inhibited by background, SES is expected to be at the level of $10^{-10}$. Currently NA62 has a sample of $1.1 \times 10^{3} K^{+} \rightarrow \pi^{+} e^{+} e^{-}$ candidates, where the decay was observed in the range $m_{e e}<140 \mathrm{MeV} / \mathrm{c}^{2}$ for the first time. SES for the decay chain $K^{+} \rightarrow \pi^{+} X, X \rightarrow e^{+} e^{-}$is around $10^{-9}$.

\section{References}

[1] T. Asaka, M. Shaposhnikov, Phys. Lett. B 620, 17 (2005)

[2] The NA62 Collaboration, JINST 12, P05025 (2017)

[3] W. A. Rolke, A. M. Lopez, Nucl. Instrum. Methods A 458, 754 (2001)

[4] The NA62 Collaboration, Phys. Lett. B 778, 137 (2018) 